\section{Calibration and validation of an optical sensor for intracellular oxygen measurements}

\author{
Dhruv Sud and Mary-Ann Mycek* \\ University of Michigan, Department of Biomedical \\ Engineering, 1101 Beal Avenue, Ann Arbor, Michigan \\ 48109-2099
}

\begin{abstract}
Calibration of fluorescent optical sensors for accurate, quantitative intracellular measurements in vivo suffers from lack of a representative medium that appropriately simulates the molecular complexity of the cytosol. We present a novel protocol for accurate intracellular oxygen sensing via fluorescence lifetime imaging microscopy (FLIM) using cell lysate-FLIM measurements to correct the in vitro calibration of a fluorescent oxygen sensor, and we describe electron paramagnetic resonance (EPR) validation studies. Lysate-FLIM studies provided biochemical information, while EPR provided a "gold standard" for intracellular oxygen estimation. Oxygen levels were evaluated in living human normal squamous and adenocarcinoma esophageal epithelial cells, and good agreement was observed between oxygen levels derived from the optical protocol and EPR. The proposed protocol introduces the concept of a living cell line as a reference for estimating unknown oxygen levels in other cell lines and accounts for high degrees of variability between different cell lines. (- 2009 Society of Photo-Optical Instrumentation Engineers. [DOI: 10.1117/1.3116714]
\end{abstract}

Keywords: fluorescence microscopy; lifetime sensing; oxygen sensing; cell culture; cellular lysis; electron paramagnetic resonance.

Paper 08357LR received Oct. 6, 2008; revised manuscript received Feb. 17, 2009; accepted for publication Feb. 17, 2009; published online Apr. 13, 2009.

Advances in high-resolution imaging capabilities and fluorescent probe development have enabled the study of physiological processes within single living cells. Intracellular monitoring of $\mathrm{pH}$, calcium, chloride, and oxygen provide an opportunity to create real-time descriptions of the biomolecular cellular state. ${ }^{1-3}$ Calibration of fluorescent sensors sensitive to these molecules is necessary for accurate quantitative results. A recurring challenge is the inability to calibrate the fluorescent probe in a medium that truly mimics the intracellular environment.

Excited-state fluorophore lifetime (and, hence, fluorescence intensity) is inherently dependent on microenvironment, which makes reproducing the intracellular molecular environment essential for accurate calibration. Toward this end, several types of solvents have been employed, including phosphate buffer saline (PBS), varied culture media, and even poisoned cells. ${ }^{2,4}$ These approaches are either simplistic or raise concern about cellular health and response. Coupled with the fact that different cell lines can vary significantly in

*Address all correspondence to: mycek@umich.edu.

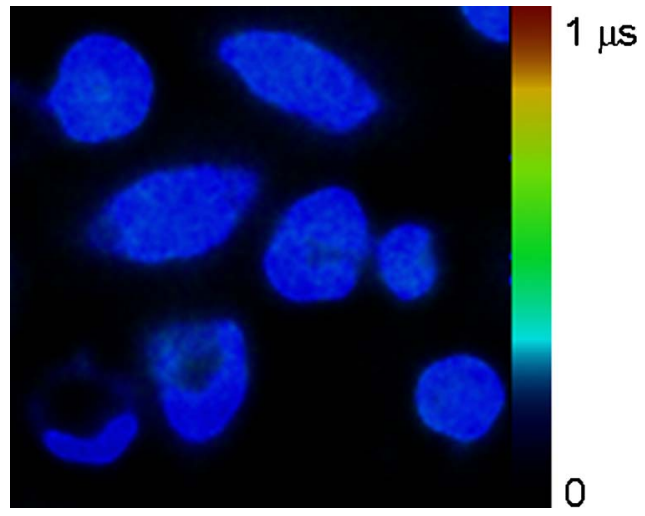

Fig. 1 Intensity-overlay FLIM image of the oxygen sensor RTDP in living cells $(\sim 10-12 \mu \mathrm{m}$ diam). RTDP lifetime maps serve as the input to the Stern-Volmer equation $\left[\mathrm{O}_{2}\right]=\left(\tau_{0} / \tau-1\right) / K_{\mathrm{q}}$ for estimation of oxygen levels.

their intracellular composition, any calibration of the fluorescent probe is unlikely to be universally applicable. The methods discussed here aim to provide a generic protocol for accurate intracellular oxygen sensing with any oxygen-sensitive fluorophore. The protocol developed employs a calibration correction derived from optical measurements on cellular lysate, which is representative of the intracellular environment and commonly used to recover enzymes and proteins in their active states.

Oxygen is the terminal electron acceptor of oxidative phosphorylation in mitochondria and a key indicator of metabolic function in aerobic cells and organisms. Information obtained from accurate oxygen estimation has been applied to study mitochondrial function, signaling pathways, effects of various stimuli, membrane permeability, disease differentiation, and to screen for new drugs. ${ }^{6-9}$ Various methods exist for estimating oxygen at different scales, whether in whole organs [magnetic resonance imaging (MRI) and nuclear magnetic resonance (NMR)], blood (absorption), tissues [electrochemical microelectrodes], or individual cells (phosphorescence, fluorescence, [electron paramagnetic resonance $(\mathrm{EPR})]^{10-13}$ Optical approaches, such as fluorescence microscopy, provide the most viable approach for intracellular oxygen sensing with high sensitivity and subcellular spatial resolution. Fluorescence lifetime-based methods additionally circumvent most complications associated with intensity-based studies, such as photobleaching, optical loss, and variable fluorophore concentration in cells. ${ }^{14}$

Figure 1 shows a representative intensity-overlay fluorescence lifetime imaging microscopy (FLIM) image of the fluorescent oxygen sensor ruthenium tris(2,2'-dipyridyl) dichloride hexahydrate (RTDP) in living cells. Such lifetime maps serve as the input to the Stern-Volmer equation for estimation of oxygen levels via collisional quenching. The oxygen sensitivity of RTDP was calibrated at $37^{\circ} \mathrm{C}$ in PBS and applied to extracellular and intracellular oxygen measurements using FLIM. ${ }^{2,15}$ Here, for the first time, lysate-FLIM studies were performed to refine this calibration and to make it sample specific. All fluorescence-based oxygen measurements were then compared to EPR.

1083-3668/2009/14(2)/020506/3/\$25.00 @ 2009 SPIE 
Table 1 Lifetime differences $\Delta \tau=\tau_{\mathrm{HET}}-\tau_{\mathrm{SEG}}$ and oxygen estimates from FLIM experiments on living cells and cellular lysates. Revised values of $\Delta \tau$ (computed as a-b) were used to correct $\left[\mathrm{O}_{2}\right]_{\mathrm{SEG}}$ levels. Corrected FLIM-derived oxygen estimates $\left[\mathrm{O}_{2}\right]_{\mathrm{FLIM}}$ were in good agreement with EPR validation measurements $\left[\left[\mathrm{O}_{2}\right]_{\text {EPR }} . K_{\mathrm{q}}\right.$ values were estimated via the Stern-Volmer equation with known lifetime and corrected oxygen values for both HET and SEG cell lines.

\begin{tabular}{ccccccc}
\hline Sample & $\begin{array}{c}\Delta \tau \\
(\mathrm{ns})\end{array}$ & $\begin{array}{c}{\left[\mathrm{O}_{2}\right]} \\
(\mu \mathrm{M})\end{array}$ & $\begin{array}{c}\text { Revised } \\
\Delta \tau(\mathrm{ns})\end{array}$ & $\begin{array}{c}{\left[\mathrm{O}_{2}\right]_{\mathrm{FLIM}}} \\
(\mu \mathrm{M})\end{array}$ & $\begin{array}{c}{\left[\mathrm{O}_{2}\right]_{\text {EPR }}} \\
(\mu \mathrm{M})\end{array}$ & $\begin{array}{c}\text { Estimated } \\
K_{\mathrm{q}}\end{array}$ \\
\hline HET cells & & 182.1 & & $182.1 \pm 9.4$ & $180.0 \pm 9.4$ & 0.0044 \\
SEG cells & $44(\mathrm{a})$ & 260.5 & 30.6 & $225.8 \pm 10$ & $210.1 \pm 8.5$ & 0.0054 \\
\hline HET-lysate & & 227 & & & & \\
& & & & & & \\
SEG-lysate & $13.4(\mathrm{~b})$ & 227 & & & & \\
\hline
\end{tabular}

Cellular lysate of HET-1 (human normal squamous esophageal epithelial cells) and SEG-1 (human Barrett's adenocarcinoma esophageal epithelial cells) were generated using the NP-40 buffer via the following protocol. Briefly, NP-40 was thawed on ice and $10 \mu \mathrm{L} / \mathrm{mL}$ of Halt Protease Inhibitor was added for inhibition of serine-, cysteine-, metallo-, and aspartic acid-proteases in addition to aminopeptidases. Because of DNA/RNA release, $50 \mu \mathrm{L} / \mathrm{mL}$ each of DNAse and RNAse was added to reduce viscosity. Cells grown in culture flasks were scraped off into PBS, centrifuged, and washed twice with ice-cold PBS. The NP-40 formulation was added in a ratio of $1 \mathrm{~mL}$ buffer $/ 10^{8}$ cells. The solution was placed on ice and vortexed for $30 \mathrm{~s}$ every $10 \mathrm{~min}$. After $30 \mathrm{~min}$, the solution was transferred to microcentrifuge tubes and run at $13,000 \mathrm{rpm}$ for $10 \mathrm{~min}$ at $4{ }^{\circ} \mathrm{C}$. This allowed the heavier debris (e.g., cell membrane components) to sediment; the clear aliquot was removed and frozen at $-80{ }^{\circ} \mathrm{C}$. For lysateFLIM experiments, $0.8 \mathrm{~mL}$ of lysate was combined with $0.2 \mathrm{~mL}$ of $5 \mathrm{mg} / \mathrm{mL}$ RTDP in a $37{ }^{\circ} \mathrm{C}$ heated dish and lifetime measurements were immediately taken, as described previously. $^{2}$

EPR oximetry is a spectroscopic technique to detect materials with unpaired electrons, such as oxygen. ${ }^{11}$ Microcrystalline $\mathrm{LiNc}-\mathrm{BuO}$ particles were suspended in $10 \mathrm{mg} / \mathrm{mL}$ of minimum essential medium (MEM). A probe sonicator was used to create particles with sizes $<200 \mathrm{~nm}$ by pulsing the sample 10 times for $30 \mathrm{~s}$ each, with 1-min cooling time (on ice) between successive pulses. The sample was placed on ice for 10-15 min to allow heavier, larger particles to settle. The supernatant liquid was then aliquoted out. For treatment with the EPR particles, both HET and SEG were grown to $70 \%$ confluence in 35-mm dishes, then suspended in $1 \mathrm{~mL}$ MEM. To this suspension, $50 \mu \mathrm{L}$ of the $\mathrm{LiNc}-\mathrm{BuO}$ suspension was added and the cells were incubated for $48 \mathrm{~h}$. The media were then replaced, the cells were resuspended, and $20 \mu \mathrm{L}$ of the suspension $(\sim 4000$ cells $)$ was drawn into a glass tube. Previous reports have indicated successful internalization of LiNc$\mathrm{BuO}$ into cells, as well as removal of remaining extracellular particles in the media by this approach. ${ }^{16}$ The tube was placed in a flat cell resonator that was carefully aligned in the microwave cavity of an EPR spectrometer (Bruker EMX). EPR spectra were acquired for both cell lines, as specified in the manufacturer's guidelines for the spectrometer (www.bruker. com). A previously reported calibration was used to convert the spectral bandwidth to oxygen levels $\left(0 \%[\mathrm{O}]_{2}=210 \mathrm{mG}\right.$ line width, $8.5 \mathrm{mG} / \mathrm{mmHg}$ oxygen sensitivity for LiNc-Buo). ${ }^{16}$

Table 1 shows the analysis of results. Both HET-1 and SEG-1 were exposed to similar oxygen levels $(227 \mu \mathrm{M}$ in solution), yet exhibited different RTDP lifetimes. Given that both cell lysates were generated in an identical fashion and that the experiments were not affected by factors such as $\mathrm{pH}$, viscosity, temperature differences, or aggregation (shown previously in Ref. 2), the differences in lysate RTDP lifetime were likely indicative of cytosolic composition and/or of greater dynamic quenching in SEG versus HET. The differences in lysate RTDP lifetime were small compared to RTDP lifetime $(\sim 13.4 / 300 \mathrm{~ns}$, or $\sim 4.5 \%)$ and were statistically significant $(p>0.05)$.

Live-cell FLIM revealed an average intracellular RTDP lifetime difference of 44 ns between HET and SEG cell lines, which led to initial, uncorrected estimates of $\left[\mathrm{O}_{2}\right]_{\text {HET }}$ $=182.1 \mu \mathrm{M}$ and $\left[\mathrm{O}_{2}\right]_{\mathrm{SEG}}=260.5 \mu \mathrm{M}$, as shown in Table 1 and reported elsewhere. ${ }^{2}$ Given that extracellular oxygen was $\sim 227 \mu \mathrm{M}$ and no known mechanisms of active oxygen transport exists in cells, it is likely that $\left[\mathrm{O}_{2}\right]_{\text {SEG }}$ was overestimated by the uncorrected calibration. Indeed, cell lysate studies indicated that the potential lifetime difference might have been overestimated by $\sim 13.4$ ns (Table 1 ). When corrected by this factor, we obtain a revised average intracellular RTDP lifetime difference $\Delta \tau=30.6 \mathrm{~ns}$ and corrected value of $\left[\mathrm{O}_{2}\right]_{\text {FLIM:SEG }}=225.8 \mu \mathrm{M}$.

EPR oximetry provided a well-established means of validating these intracellular oxygen studies. Lysate studies on the experimental probe $\mathrm{LiNc}-\mathrm{BuO}$ indicated little or no effect of the intracellular environment. As shown in Table 1 data in gray boxes), intracellular oxygen levels measured via EPR on living HET cells $\left(\left[\mathrm{O}_{2}\right]_{\mathrm{EPR}: \mathrm{HET}}=180.0 \mu \mathrm{M}\right)$ were very close to the values estimated via RTDP FLIM maps of living HET cells. Also (data in gray boxes), intracellular oxygen levels measured via EPR on living SEG cells ( $\left[\mathrm{O}_{2}\right]_{\text {EPR:SEG }}$ $=210.1 \mu \mathrm{M}$ ) were close to the values estimated via RTDP FLIM maps of living SEG cells that were corrected using 


\section{JBO LETTERS}

correction values obtained from the cell lysate studies described above. We note that the trend of $\left[\mathrm{O}_{2}\right]_{\mathrm{SEG}}>\left[\mathrm{O}_{2}\right]_{\mathrm{HET}}$ was maintained, despite some expected variations due to the differences between fluorescence imaging and EPR spectroscopic methods (e.g., the use of adherent vs. suspended cell samples).

These results suggest a novel protocol for intracellular oxygen sensing in living cells by using a reference cell line with known oxygenation. In the study reported here, that purpose was served by the HET cells due to their stable oxygen levels across both fluorescence and EPR studies. However, any established cell line could be used in this role. For example, commercially available Chinese Hamster Ovary (CHO) cells have been used for EPR oxygenation studies with reproducible results $\left(\left[\mathrm{O}_{2}\right]_{\mathrm{CHO}}=180 \pm 9.3 \mu \mathrm{M}\right){ }^{6}$

The protocol, extensible to any oxygen-sensitive fluorophore, would involve measuring $\tau_{0}$ for the fluorophore in solution. Both the reference and the experimental cell lines would then be incubated with the probe to estimate oxygen levels, which could be corrected via lysate-FLIM studies. Finally, corrected lifetime and oxygen values could be used to compute the Stern-Volmer quenching constant $K_{\mathrm{q}}$ that is specific to the experimental cell line and valid for all future experiments without the need for any additional corrections.

Linear subtraction of lifetimes obtained from the RTDPcell and RTDP-lysate studies is a simplified assumption made due to the unknown nature of the biochemical component that quenches RTDP lifetime. Future work in this regard could involve use of more accurate and complicated mathematical models for correcting measured lifetime values. Compared to previous calibration efforts for intracellular oxygen sensing, the validated FLIM-based approach described here has the advantages of using a living reference biological sample, accounting for specific intracellular biochemical information, and enabling accurate, quantitative mapping of intracellular oxygen in vivo.

\section{Acknowledgments}

The authors thank Dr. Periannan Kuppusamy (Ohio State University) for providing the $\mathrm{LiNc}-\mathrm{BuO}$ particulates and Arjun Khullar (University of Michigan) for early efforts on the EPR project. This work was supported by a grant from the National Institutes of Health Grant No. NIH CA-114542 (to M.-A.M.).

\section{References}

1. C. Balut, M. vandeVen, S. Despa, I. Lambrichts, M. Ameloot, P. Steels, and I. Smets, "Measurement of cytosolic and mitochondrial $\mathrm{pH}$ in living cells during reversible metabolic inhibition," Kidney Int. 73, 226-232 (2008).

2. D. Sud, W. Zhong, D. G. Beer, and M. A. Mycek, "Time-resolved optical imaging provides a molecular snapshot of altered metabolic function in living human cancer cell models," Opt. Express 14, 44124426 (2006).

3. P. Rohrbach, O. Friedrich, J. Hentschel, H. Plattner, R. H. Fink, and M. Lanzer, "Quantitative calcium measurements in subcellular compartments of Plasmodium falciparum-infected erythrocytes," J. Biol. Chem. 280, 27960-27969 (2005).

4. C. J. Grauw and H. C. Gerritsen, "Fluorescence lifetime imaging of oxygen in dental biofilm," Proc. SPIE 4164, 70-78 (2003).

5. T. C. O'Riordan, A. V. Zhdanov, G. V. Ponomarev, and D. B. Papkovsky, "Analysis of intracellular oxygen and metabolic responses of mammalian cells by time-resolved fluorometry," Anal. Chem. 79, 9414-9419 (2007).

6. N. Khan, J. Shen, T. Y. Chang, C. C. Chang, P. C. Fung, O. Grinberg, E. Demidenko, and H. Swartz, "Plasma membrane cholesterol, a possible barrier to intracellular oxygen in normal and mutant $\mathrm{CHO}$ cells defective in cholesterol metabolism," Biochemistry 42, 23-29 (2003).

7. C. Simpkins, S. Balderman, and E. Mensah, "Mitochondrial oxygen consumption is synergistically inhibited by metallothionein and calcium," J. Surg. Res. 80, 16-21 (1998).

8. H. M. Swartz, "Using EPR to measure a critical but often unmeasured component of oxidative damage, Oxygen," Antioxidants Redox Signal. 6, 677-686 (2004).

9. H. M. Swartz, N. Khan, J. Buckey, R. Comi, L. Gould, O. Grinberg, A. Hartford, H. Hopf, H. G. Hou, E. Hug, A. Iwasaki, P. Lesniewski, I. Salikhov, and T. Walczak, "Clinical applications of EPR: overview and perspectives," NMR Biomed. 17, 335-351 (2004).

10. S. M. Evans, K. D. Judy, I. Dunphy, W. T. Jenkins, P. T. Nelson, R. Collins, E. P. Wileyto, K. Jenkins, S. M. Hahn, C. W. Stevens, A. R. Judkins, P. Phillips, B. Geoerger, and C. J. Koch, "Comparative measurements of hypoxia in human brain tumors using needle electrodes and EF5 binding," Cancer Res. 64, 1886-1892 (2004).

11. B. Gallez, and H. M. Swartz, "In vivo EPR: when, how and why?" NMR Biomed. 17, 223-225 (2004).

12. H. M. Swartz, and J. Dunn, "The difficulties in comparing in vivo oxygen measurements: Turning the problems into virtues," in Oxygen Transport to Tissue XXVI Vol. 566, pp. 295-301 (2005).

13. W. Zhong, P. Urayama, and M. A. Mycek, "Imaging fluorescence lifetime modulation of a ruthenium-based dye in living cells: the potential for oxygen sensing," J. Phys. D 36, 1689-1695 (2003).

14. P. K. Urayama, W. Zhong, J. A. Beamish, F. K. Minn, R. D. Sloboda, K. H. Dragnev, E. Dmitrovsky, and M. A. Mycek, "A UV-visible fluorescence lifetime imaging microscope for laser-based biological sensing with picosecond resolution," Appl. Phys. B 76, 483-496 (2003).

15. D. Sud, G. Mehta, K. Mehta, J. Linderman, S. Takayama, and M. A. Mycek, "Optical imaging in microfluidic bioreactors enables oxygen monitoring for continuous cell culture," J. Biomed. Opt. 11, 050504 (2006).

16. V. K. Kutala, N. L. Parinandi, R. P. Pandian, and P. Kuppusamy, "Simultaneous measurement of oxygenation in intracellular and extracellular compartments of lung microvascular endothelial cells," Antioxid. Redox Signal 6, 597-603 (2004). 